OPEN ACCESS

Edited by:

Ana Rotter,

National Institute of Biology (NIB),

Slovenia

Reviewed by:

Lingling Feng,

Central China Normal University,

China

Kathryn Coyne,

University of Delaware, United States

${ }^{*}$ Correspondence:

Shuangfei $L$

szu_sfl@163.com

Specialty section:

This article was submitted to Marine Biotechnology,

a section of the journal

Frontiers in Marine Science

Received: 06 March 2021

Accepted: 10 May 2021

Published: 11 June 2021

Citation:

Ouyang L, Liu Y, Chen H, Zaynab M, Yang $X$, Wang S and Li S (2021) Encapsulation and Algicidal Properties of Fermentation Products

From Vibrio brasiliensis $\mathrm{H} 115$.

Front. Mar. Sci. 8:676913.

doi: 10.3389/fmars.2021.676913

\section{Encapsulation and Algicidal Properties of Fermentation Products From Vibrio brasiliensis H115}

\author{
Liao Ouyang ${ }^{1}$, Yan Liu' ${ }^{2}$, Huirong Chen ${ }^{1}$, Madiha Zaynab' ${ }^{1}$, Xuewei Yang ${ }^{1}$, Shilin Wang ${ }^{1}$ \\ and Shuangfei $\mathrm{Li}^{1 *}$ \\ 'Shenzhen Key Laboratory of Marine Bioresource \& Eco-Environmental Sciences, College of Life Sciences \\ and Oceanography, Shenzhen University, Shenzhen, China, ${ }^{2}$ School of Business Administration, Heyuan Polytechnic, \\ Heyuan, China
}

Algicidal bacteria offer an eco-friendly and promising approach for controlling harmful algae blooms (HABs). In this study, repeated batch fermentation of immobilized algicidal bacterium Vibrio brasiliensis $\mathrm{H} 115$ was preformed to enhance the productivity of the algicidal compounds. The highest algicidal efficiency of the fermentation products against Akashiwo sanguinea (100\%) was achieved when the fermentation time was decreased from 24 to $14 \mathrm{~h}$. The cell-free fermentation broth was then spray-dried and floating microcapsules were prepared from the dried powder. The optimum preparation conditions for floating microcapsules were: sodium alginate (SA), 3\%; $\mathrm{CaCO}_{3}$ : $\mathrm{SA}$ (mass ratio), 3:4; $\mathrm{CaCl}_{2}, 3 \%$; citric acid, 4\%; ethylcellulose, 2\%; crosslinking time, 30 min. Under the optimal conditions, the floating microcapsules displayed efficient $A$. sanguinea cell lysis ability and the algicidal efficiency increased from 10.62\% (4 h) to 100\% (24 h). These results suggest that the floating microcapsules could potentially be practically used for controlling the outbreaks of $A$. sanguinea.

Keywords: red tides, algicidal bacteria, fermentation, floating microcapsules, Vibrio brasiliensis

\section{INTRODUCTION}

Red tides have attracted worldwide attention as they pose significant threats to fishery resources, marine ecosystems and human health (Anderson, 2009). Biological methods have been broadly investigated for the control of red tides as it has obvious advantages over physical and chemical methods (Li et al., 2016). During the last decades, algicidal bacteria, which are capable of algal cell lysis or growth inhibition have been isolated from lakes, estuaries, and seas. They offer a promising and eco-friendly biological approach for the control of harmful algae blooms (HABs) (Tian et al., 2012; Lu et al., 2016; Zheng et al., 2018).

Various isolated algicidal bacteria have been preliminarily studied in the lab to confirm their algal lysis ability (Tian et al., 2012; Zhang et al., 2018; Tarazona-Janampa et al., 2020). However, the practical application of algicidal bacteria in controlling algal blooms is challenged in many ways. First, the complex and unstable environment in the natural ecosystem, such as low temperature and competition from other bacteria, may inhibit the growth of the algicidal bacteria and reduce their algicidal activity (Kang et al., 2012). In addition, the algicidal activity of such bacteria is species-specific. Nevertheless, algicidal bacteria and their associated metabolic products might result in a negative impact on other species and the ecosystem (Kang et al., 2007; 
Jung et al., 2010). The addition of Pseudomonas fluorescens SK09 in a large-scale mesocosm natural bloom of Stephanodiscus hantzschii changed the composition of the phytoplankton community (Jung et al., 2010). Therefore, for practical and commercial application of algicidal bacteria, the development of strategies that are capable of sustaining the algicidal activity as well as minimizing their adverse effects is essential.

Most of the algicidal bacteria attack the target algae in an indirect mode by secreting active algicidal compounds (such as agarase, amino-peptidase, lipase, proteins, alkaline phosphatase and enzymes) to the surrounding medium (Wang M. et al., 2020). The synthesis of these active compounds is mainly based on bacterial metabolism. Studies have shown that the productivity of secondary metabolites of microorganisms can be enhanced during repeated fermentations (Li H.-X. et al., 2015; Wang et al., 2016; Xu et al., 2020). Thus, fermentation is an essential process for the large-scale production of the algicidal compounds ( $\mathrm{Hu}$ et al., 2020). The fermentation products of algicidal bacteria can possibly be considered as biological agents for the control of HABs. However, limited studies have been conducted regarding the potential application of fermentation products. It was reported that the fermentation products from Bacillus cereus strain JZBC1 were capable of algicidal activity against Scrippsiella trochoidea, Prorocentrum micans, and Peridinium umbonatum (Hu et al., 2020). Our previous study also confirmed the algicidal activity of the spray-dried algicidal broth against Akashiwo sanguinea (Wang Y. et al., 2020). Bacillamide secreted by Bacillus sp. SY-1 (Jeong et al., 2003) and the pigment deinoxanthin, isolated from Deinococcus sp. Y35 (Li Y. et al., 2015) had algicidal effect on Alexandrium tamarense and Cochlodinium polykrikoides, respectively. Compared to bacterial cells, the algicidal powder is capable of minimizing the ecological burden of direct bacterial cell addition. So it is necessary to seek a method that enables the direct application of algicidal powder to control HABs.

Immobilization is a long-established method used for increasing the packing yield of enzymes, microorganisms, organelles, and plant/animal cells in the biotechnology processes (Meleigy and Khalaf, 2009; Ma et al., 2015; Ahmad et al., 2020). As one of the widely used methods of immobilization, microcapsule technology has been widely applied in the fields of medicine, pesticides and food (Mori et al., 1972; Fukumori et al., 1988). In terms of algicidal bacteria and their corresponding metabolites, Pseudomonas fluorescens SK09 was successfully immobilized to control Stephanodiscus hantzschii blooms (Jung et al., 2013), Shewanella sp. IRI-160 immobilized in alginate beads were used to control the growth of Karlodinium veneficum and Prorocentrum minimum (Wang and Coyne, 2020). Alcaligenes aquatilis F8 immobilized with wheat bran and sodium alginate (SA) also showed algicidal activity against Microcystis aeruginosa (Sun et al., 2015). Immobilization of algicidal bacteria has been shown to increase the cell density and the corresponding algicidal activity. However, to date, microcapsules containing algicidal powder have not been reported. Because HABs occurs on the water surface, floating microcapsules containing algicidal powder are proposed as a promising way to control the HABs. SA is a widely used safe biodegradable polymer for encapsulation of cells, microbes, and drugs (Nochos et al., 2008; He et al., 2015). Ethylcellulose (EC) is a water-insoluble polymer with a low density and has been studied for the encapsulation and controlled release of drugs (Yang et al., 2001; Prasertmanakit et al., 2009). Furthermore, it can enhance the mechanical strength of the microcapsules and enables effective wrapping of $\mathrm{CO}_{2}$ and thus enhancing the floating ability (Yang and Wang, 2014). The encapsulation of algicidal powder using SA and EC was proposed in this study, as it can potentially delay the release rate of algicidal compounds, thus effectively extending its shelf life with reduced side effects on the ecosystems.

The algicidal bacterium Vibrio brasiliensis $\mathrm{H} 115$ used in this study was isolated from Dameisha Bay (Shenzhen, China) and was confirmed to have algicidal activity against $A$. sanguinea in an indirect mode. The bacteria itself cannot lyse algal cells, while cell-free supernatants of the fermentation broth were responsible for the lysis of the algal cells (unpublished data). In this study, batch fermentation was conducted for the immobilized Vibrio brasiliensis $\mathrm{H} 115$ to generate algicidal compounds. Then, the cell-free fermentation broth containing algicidal compounds was spray-dried and used for the preparation of floating microcapsules. Sodium alginate (SA) and ethylcellulose (EC) were used as wall material and coating material for the floating microcapsules, respectively, and $\mathrm{CaCO}_{3}$ was used as $\mathrm{CO}_{2}$ gasforming agent. The composition of floating microcapsules was optimized using single-factor effect experiments. Furthermore, the algicidal activity of the optimal floating microcapsules against $A$. sanguinea was determined. Overall, the results of this study provide support for the potential application of algicidal powder-based floating microcapsules on preventing A. sanguinea blooms.

\section{MATERIALS AND METHODS}

\section{Algal Culture and Strain}

The algicidal bacterium Vibrio brasiliensis H115 was isolated from the seawater of Dameisha Bay (Shenzhen, China), and subsequently cultured in $2216 \mathrm{E}$ medium ( $5 \mathrm{~g} / \mathrm{L}$ peptone, $1 \mathrm{~g} / \mathrm{L}$ yeast extract, $0.1 \mathrm{~g} / \mathrm{L}$ ferric phosphate, $\mathrm{pH}$ 7.6-7.8) for $24 \mathrm{~h}$ $\left(25^{\circ} \mathrm{C}, 200 \mathrm{rpm}\right)$.

Akashiwo sanguinea used in this study was provided by the Algal Culture Collection of the Institute of Hydrobiology at Jinan University (Guangzhou, China). The culture was maintained in modified f/2 medium (Lananan et al., 2013; Zhang et al., 2014) at $20 \pm 2{ }^{\circ} \mathrm{C}$ with light (3500 l×, 12:12 light-dark cycle) (Sun et al., 2016). The algal culture was incubated for $24 \mathrm{~h}$ to achieve its exponential growth phase before use.

\section{Immobilization of Vibrio brasiliensis H115 and Repeated Batch Fermentation}

The preparation of immobilized bacteria was conducted as reported previously with slight modifications (Idris and Suzana, 2005). Briefly, Vibrio brasiliensis H115 culture (10 mL) was centrifuged $(8,000 \mathrm{rpm}, 10 \mathrm{~min})$ and the collected pellets were mixed thoroughly with $3 \%(\mathrm{~m} / \mathrm{v})$ sterilized SA (Solarbio, Beijing, China) solution $(10 \mathrm{~mL})$. The mixture was then dropped into a 
beaker with $4 \%(\mathrm{~m} / \mathrm{v}) \mathrm{CaCl}_{2}$ (Macklin, Shanghai, China) using a pipette with sterile tips $(1 \mathrm{~mL})$. The solution was stirred slowly for $24 \mathrm{~h}$ at $4^{\circ} \mathrm{C}$ to complete the bead formation process. The cell density in the bead was $7.4 \times 10^{8} \mathrm{cell} / \mathrm{g}$. The beads were washed with sterile water and stored at $4^{\circ} \mathrm{C}$ before use.

The initial fermentation was conducted by inoculating $1 \mathrm{~g}$ of the immobilized beads into a flask $(\mathrm{n}=3)$ with $100 \mathrm{~mL}$ of modified 2216E medium (with an extra $10 \mathrm{~g} / \mathrm{L}$ of sorbitol and $20 \mathrm{~g} / \mathrm{L}$ of peptone) and incubating at $40^{\circ} \mathrm{C}$ and $200 \mathrm{rpm}$ for up to $26 \mathrm{~h}$. The fermentation broth was collected at different time points $(0,4,8,14,18,22,24$, and $26 \mathrm{~h})$ for the detection of $\mathrm{OD}_{600}$ and then centrifuged $(10,000 \mathrm{~g}, 10 \mathrm{~min})$. The $\mathrm{OD}_{600}$ in the fermentation broth was used as a proxy for the cell density of H115 in the beads. The supernatants were collected and filtered to get the cell-free fermentation broth. The algicidal efficiency of the cell-free fermentation broth against $A$. sanguinea was detected by co-incubating the cell-free fermentation broth $(1 \mathrm{~mL})$ with A. sanguinea $(100 \mathrm{~mL})$ for $10 \mathrm{~min}$. A control experiment was added by co-incubating $1 \mathrm{~mL}$ of sterile modified $2216 \mathrm{E}$ with $100 \mathrm{~mL} \mathrm{~A}$. sanguinea for $10 \mathrm{~min}$ to test if the modified $2216 \mathrm{E}$ medium has any effect on A. sanguinea. Algicidal efficiency was calculated as described by Zhang et al. (2018):Algicidal efficiency $(\%)=100 \times\left(\mathrm{N}_{0}-\mathrm{Nt}\right) / \mathrm{N}_{0}$, where $\mathrm{N}_{0}$ and $\mathrm{Nt}$ represent the cell density of $A$. sanguinea measured at $0 \mathrm{~h}$ and different sampling time $(\mathrm{t})$, respectively. Bioassays were conducted in triplicate. The cell number of the $A$. sanguinea was counted using a microscope (Olympus IX51, Tokyo, Japan) with a hemocytometer after staining with Lugol's reagent (Wang Y. et al., 2020).

After the initial fermentation, the immobilized beads were collected and washed with sterile distilled water three times before inoculating into $100 \mathrm{~mL}$ of optimized $2216 \mathrm{E}$ medium for repeated batch fermentations. Repeated batch fermentation was conducted three times as above to determine the algicidal activity of the beads and evaluate their stability.

\section{Preparation of the Fermentation Broth Powder and Floating Microcapsules}

After $14 \mathrm{~h}$ of fermentation, the fermentation broth was collected and centrifuged $(10,000 \mathrm{~g}, 10 \mathrm{~min})$, the supernatants were collected and filtered to get the cell-free supernatants. The cellfree supernatants were sprayed $(5 \mathrm{r} / \mathrm{min})$ at $180^{\circ} \mathrm{C}$ using a spray drier (Bioq-8005, Huihetang Bioengineering Equipment Co., China) to get the powder of fermentation broth. The powder was then added into $100 \mathrm{~mL}$ A. sanguinea culture at different concentrations $(0.1-1.5 \mathrm{~g} / \mathrm{L})$ and the algicidal efficiency was calculated after $24 \mathrm{~h}$ exposure. A. sanguinea culture without the addition of powder was set as the control and three replicates were conducted. The cell number of the A. sanguinea was counted at 0 and $24 \mathrm{~h}$ as above to calculate the algicidal efficiency.

The dried powder was then used for the preparation of floating algicidal microcapsules. The preparation of the floating microcapsules was modified from the previous study (Yang and Wang, 2014). Firstly, solution A (100 mL) was prepared by mixing $\mathrm{CaCO}_{3}$ (Macklin, Shanghai, China) with 2\% of SA (3:4 ratio) and solution $\mathrm{B}(100 \mathrm{~mL})$ was made by mixing $3 \%$ citric acid (Macklin, Shanghai, China) with $3 \% \mathrm{CaCl}_{2}$. The dried powder was added into solution A with a concentration of $1.7 \mathrm{~g} / \mathrm{L}$ and mixed thoroughly, the mixture was then dropped into a beaker with solution B using a pipette with sterile tips $(1 \mathrm{~mL})$. The solution was then stirred $(200 \mathrm{rpm})$ to obtain the SA beads. The SA beads were washed with sterile water three times and airdried. The SA beads were then dispersed in ethylcellulose (EC, M70, Macklin, Shanghai, China) solution (2\%, w/v) and stirred for $20 \mathrm{~min}$ to acquire EC coating. The beads were then washed with ethanol $(100 \%)$ once and then washed with sterile water three times. The SA-EC beads were then dried at $40^{\circ} \mathrm{C}$ and stored at $4^{\circ} \mathrm{C}$ before use.

\section{Optimization of the SA-EC Microcapsules}

To determine an optimum preparation condition for the SA-EC microcapsules, single factor effect experiments were conducted to examine the effect of different factors on the physical characteristics of the SA-EC microcapsules. The microcapsules were prepared with different concentrations of SA (1.0, 2.0, 3.0, 4.0, and 5.0\%), $\mathrm{CaCO}_{3}$ : SA ratios (0, 1:4, 1:2, 3:4, and $1: 1)$, concentrations of $\mathrm{CaCl}_{2}(1.0,2.0,3.0,4.0$, and $5.0 \%)$, concentrations of citric acid $(1.0,2.0,3.0,4.0$, and $5.0 \%)$, concentrations of EC $(0,1.0,2.0,3.0,4.0$, and 5.0\%), and different crosslinking time $(10,20,30,40$, and $50 \mathrm{~min})$. When conducting the single factor effect experiments, except for the variables, the basic experimental conditions were $3 \% \mathrm{SA}, \mathrm{CaCO}_{3}$ : $\mathrm{SA}$ of $3: 4,3 \%$ $\mathrm{CaCl}_{2}, 3 \%$ citric acid, $2 \% \mathrm{EC}$, and crosslinking for $30 \mathrm{~min}$.

The diameter of the microcapsules was obtained by calculating the average diameter of fifty microcapsules, which was measured with a Vernier caliper (He et al., 2015).

To estimate the floating ability of the microcapsules, fifty microcapsules were added into $100 \mathrm{~mL}$ of seawater and incubated with shaking (50 rpm) for 4 days, and the number of the floating microcapsules was counted for the determination of floating ability.

Crystal violet solution was used as a middle marker to assess the permeability of the microcapsules. Approximately, $0.7 \mathrm{~g}$ of microcapsules were immersed in $50 \mathrm{~mL}$ of crystal violet solution (with an absorbance of about 1.0) and incubated for $4 \mathrm{~h}$ $(n=3)$. Samples $(3 \mathrm{~mL})$ were taken at 0 and $4 \mathrm{~h}$ to measure the crystal violet absorbance $\left(\mathrm{A}_{570}\right)$ using a spectrophotometer (UV-8000S, METASH, Shanghai, China). The permeability of beads was estimated based on the difference between the two OD values as follows: Permeability $(\%)=100 \times\left(\mathrm{A}_{0}-\mathrm{A}\right) / \mathrm{A}_{0}$, where $A_{0}$ and $A$ represent the absorbance of crystal violet solution measured at 0 and $4 \mathrm{~h}$, respectively. All experiments were conducted in triplicate.

\section{Algicidal Activity of the SA-EC Microcapsules}

The algicidal activity of the optimized SA-EC microcapsules was tested by inoculating $1 \mathrm{~g}$ of microcapsules into $100 \mathrm{~mL}$ of A. sanguinea culture. The concentration of algicidal powder in the microcapsules was $1.7 \mathrm{~g} / \mathrm{L}$. Samples were collected at 4, 8, 16, and $24 \mathrm{~h}$ to count the algal cell numbers and calculate the algicidal efficiency. Microcapsules without powder were used as control. 
The maximum photosynthetic quantum yield (QY) of PSII $(\mathrm{Fv} / \mathrm{Fm})$ and the relative electron transport rate (rETR) were also analyzed using a chlorophyll fluorometer (Water-PAM, WALZ, Germany). Prior to fluorescence measurement, samples were dark-acclimated at room temperature for $20 \mathrm{~min}$. The untreated cell-free $A$. sanguinea culture was used as blank when determining the Fv/Fm and rETR.

\section{Statistical Analyses}

Statistical analyses were performed in R (v. 3.6.0). At least three replicates were conducted in all experiments, data were presented as mean \pm standard error. Significant changes $(p<0.05)$ of algicidal efficiency over time/concentration of algicidal powder were analyzed by one-way ANOVA. To test the significant difference $(p<0.05)$ of measured variables (algicidal efficiency, Fv/Fm and rETR) between control and treatment groups over time, data were analyzed by two-way ANOVA with time and treatment as independent variables and followed by Tukey's HSD post hoc tests.

\section{RESULTS AND DISCUSSION}

\section{Fermentation of the Immobilized Strain H115 and Its Algicidal Efficiency}

Repeated batch fermentation of immobilized bacteria has been widely applied for the production of secondary metabolites. Studies have shown that the productivity of secondary metabolites of microorganisms, such as penicillin (Rani et al., 2004), gibberellic acid (Meleigy and Khalaf, 2009), cyclosporin A (Suvase et al., 2010) and lovastatin (Porcel et al., 2008) can be enhanced during repeated fermentations. In this study, the algicidal activity of the cell-free fermentation broth was significantly improved using repeated batch fermentation. The algicidal efficiency of the initial fermentation was compared to the repeated batch fermentation of the immobilized strain H115 (Figure 1). Figure 1A shows the growth of H115 in the fermentation broth during the initial fermentation and the corresponding algicidal activity of the cell-free fermentation broth. The growth of H115 in the fermentation broth was used as a proxy for the cell density of H115 in the beads. Results showed that both $\mathrm{OD}_{600}$ and algicidal efficiency did not change significantly at the first $8 \mathrm{~h}(p=0.33$ and 0.40 , respectively), indicating a lag phase. The $\mathrm{OD}_{600}$ of fermentation broth increased significantly $(p<0.05)$ and reached $4.37 \pm 0.02$ at $24 \mathrm{~h}$, while the algicidal efficiency reached $100 \%$ (Figure 1A). The lag phase in the initial fermentation can be explained by the direct addition of immobilized beads without pre-activation. For the repeated fermentation, the average $\mathrm{OD}_{600}$ increased significantly from $0.43 \pm 0.01$ to $4.35 \pm 0.08$ in $16 \mathrm{~h}(p<0.05$, Figure $1 \mathrm{~B})$. The average algicidal efficiency of cell-free fermentation broth increased significantly $(p<0.05)$, and an algicidal efficiency of $100 \%$ was reached at $14 \mathrm{~h}$ (Figure 1B). To test the effect of modified 2216E medium on A. sanguinea, a control experiment was conducted by inoculating $1 \mathrm{~mL}$ of sterile modified $2216 \mathrm{E}$ with $100 \mathrm{~mL} \mathrm{~A}$. sanguinea for $10 \mathrm{~min}$. Results showed that the number of the algal cells did not change significantly before and after co-incubation with modified $2216 \mathrm{E}$ medium $(p=0.80)$,
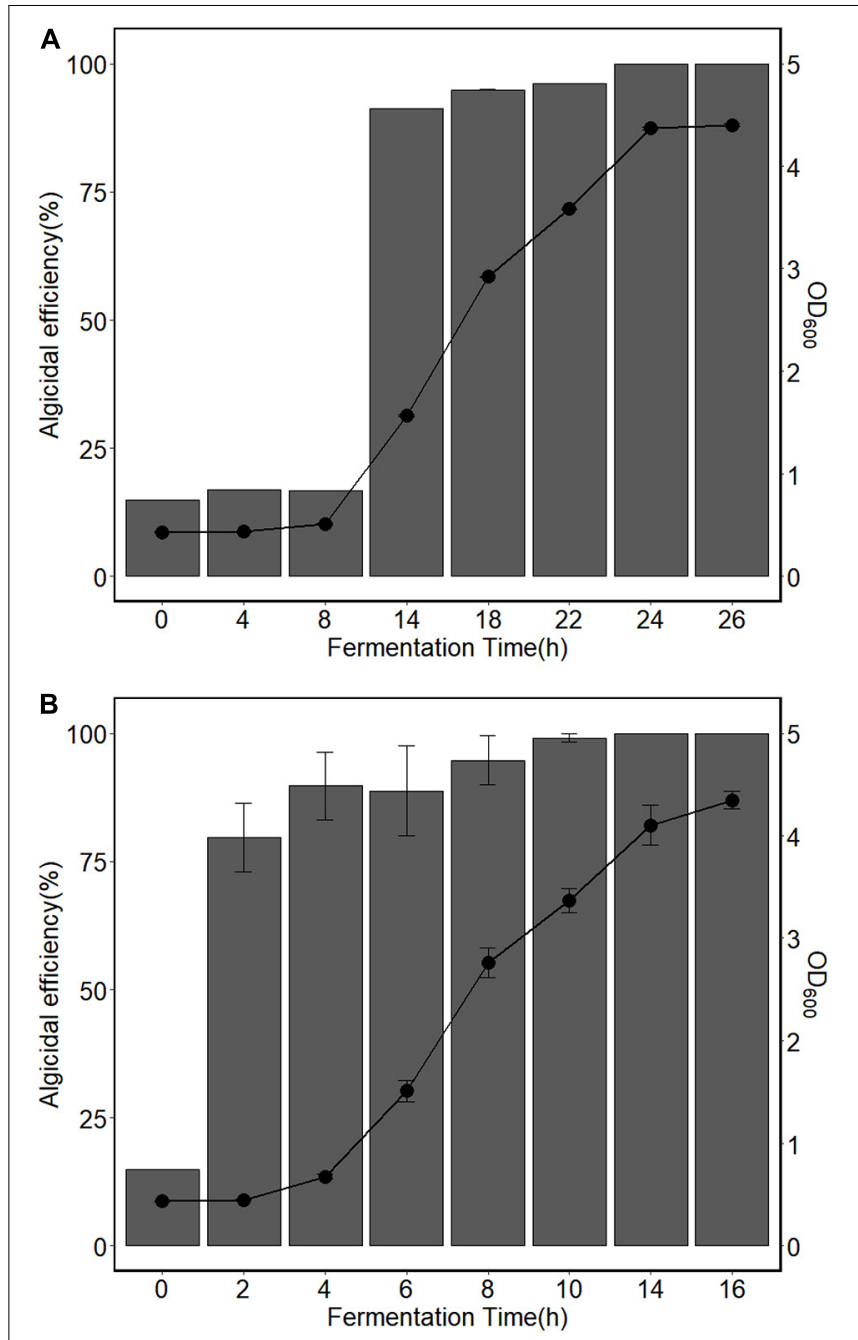

FIGURE 1 | Cell concentrations of $\mathrm{H} 115$ in fermentation broth and algicidal efficiency of cell-free fermentation broth during (A) initial fermentation and (B) repeated batch fermentation. Data for panel $\mathbf{B}$ ) was generated from the average of all three repeated batch fermentations. Bars represent the algicidal efficiencies and as indicated on the left $y$-axis. Points represent $\mathrm{OD}_{600}$ and as indicated on the right $y$-axis. Values are means \pm s.e, $n=3$.

indicating that the modified $2216 \mathrm{E}$ medium has no algicidal effect on $A$. sanguinea. These results indicated that the fermentation time of immobilized beads can be greatly shortened during repeated batch fermentations, which is of great significance for increasing the productivity of the algicidal compounds.

\section{Fermentation Powder and Its Encapsulation}

The cell-free fermentation broth of H115 was collected and spray dried to get the fermentation powder. Figure 2 shows that the algicidal efficiency increased significantly with an increase in the concentration of fermentation powder $(p<0.05)$ at the concentration ranges of $0-1.0 \mathrm{~g} / \mathrm{L}$. The maximum algicidal efficiency $(100 \%)$ was achieved when the concentration of the fermentation powder reached $1.0 \mathrm{~g} / \mathrm{L}$ of algal culture. This could be explained by the dose-response relationship between algicidal 


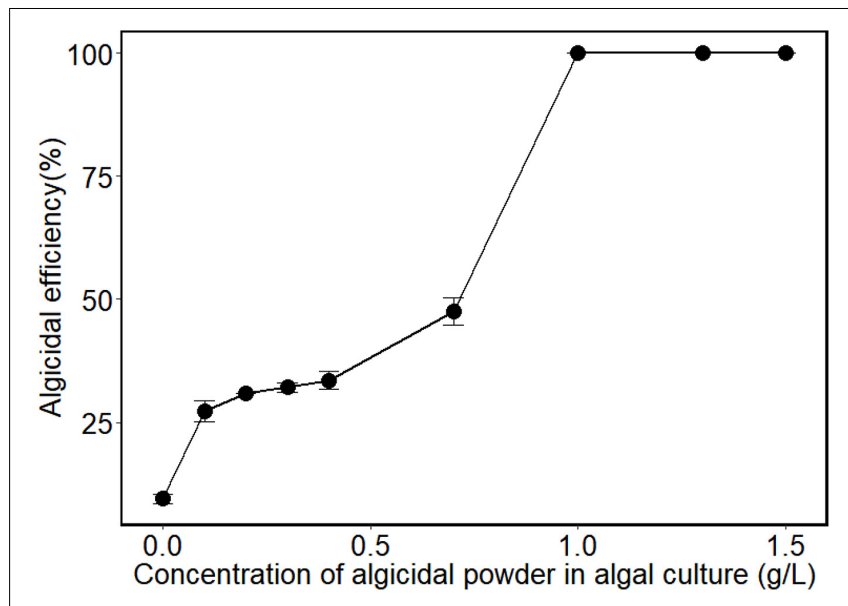

FIGURE 2 | Algicidal efficiency of the algicidal powder against $A$. sanguinea at different concentrations (Algicidal powder was made from the cell-free fermentation broth after $14 \mathrm{~h}$ of fermentation). Values are means \pm s.e, $n=3$.

compounds and algae cells, that is the maximal effect of algal cell lysis can be achieved at a certain dose of algicidal compounds (Tilney et al., 2014; Wang Y. et al., 2020). A similar trend was found in the study of Wang Y. et al. (2020), where the $\mathrm{EC}_{50}$ and $\mathrm{EC}_{90}$ values of algicidal compounds to $A$. sanguinea were 0.68 and $1.43 \mathrm{~g} / \mathrm{L}$, respectively. Karlodinium veneficum also exhibited a typical dose response in photochemical inhibition and cell density with increasing IRI-160AA, with an average $\mathrm{EC}_{50}$ of 7.9\% (v/v) IRI-160AA (Tilney et al., 2014). Using fermentation powder as core material, floating microcapsules were successfully prepared with SA as wall material and EC as the coating material. Floating microcapsules have been widely applied for the encapsulation and controlled release of the drugs in drug delivery systems (Ma et al., 2008; Ahmed et al., 2016; Selvakumaran et al., 2016). However, studies that explore the potential application of floating microcapsules in other areas are sparse. Yang and Wang prepared sodium alginate/hydroxypropyl methylcellulose (SA/HPMC) microcapsules that can float in hai-hong wine (Yang and Wang, 2014). Immobilized beads with algicidal bacteria (Kang et al., 2012; Jung et al., 2013; Wang and Coyne, 2020) were shown to improve the algicidal efficiency for the potential control of HABs. However, to the best of our knowledge, this is the first report of the preparation of floating microcapsules with active algicidal compounds. When compared with the algicidal bacterial based immobilized beads, the floating microcapsules containing algicidal powder have advantages. The active algicidal compounds in microcapsules released slowly and sustainedly from the microcapsules, which enables efficient contact between the active algicidal compound and algae.

\section{Effect of Different Factors on the Microcapsules \\ Concentration of SA}

Different concentrations of SA $(1,2,3,4$, and 5\%) were investigated for the preparation of floating microcapsules. Results showed that the diameter of the microcapsules increased with increasing SA concentration, while the permeability decreased from 84.23 to $71.86 \%$. This finding is consistent with the study of Li et al. (2019). The maximum floating ability (70.83\%) was achieved at a SA concentration of 3\% (Table 1). Alginates are anionic compounds and form hydrogels in the presence of $\mathrm{Ca}^{2+}$ (Nochos et al., 2008). A low concentration of SA results in a flaccid microstructure for the microcapsules, thus the generated gas cannot be efficiently wrapped which resulted in a low floating ability. With an increase in SA concentration, the ionic crosslinking between $\mathrm{Ca}^{2+}$ and SA resulted in a more compact structure of the alginate capsules (Li et al., 2019). However, microcapsules with a rigid microstructure could prevent $\mathrm{H}^{+}$ from entering into the solution and reacting with $\mathrm{CaCO}_{3}$ to make sufficient $\mathrm{CO}_{2}$ gas, thus reducing the floating ability. As the floating ability is a key indicator for the microcapsules, $3 \%$ was chosen as the optimum concentration for SA.

\section{Mass Ratio of $\mathrm{CaCO}_{3}$ : SA}

$\mathrm{CaCO}_{3}$ has been successfully used for the preparation of floating alginate beads (Choi et al., 2002; Ma et al., 2008) and the floating drug delivery system (Selvakumaran et al., 2016). As a $\mathrm{CO}_{2}$ gasforming agent, the concentration of $\mathrm{CaCO}_{3}$ plays a key role in the floating ability of microcapsules. During the formation of microcapsules, $\mathrm{CO}_{3}{ }^{2-}$ reacts with citric acid to produce $\mathrm{CO}_{2}$. The evolving gas permeates through the alginate leaving gas bubbles or pores (Choi et al., 2002). As seen in Table 1, negligible floating ability $(6.06 \%)$ was observed without the addition of $\mathrm{CaCO}_{3}$. The floating ability of the microcapsules increased as the relative content of $\mathrm{CaCO}_{3}$ increased, and the maximum floating ability (70.37\%) was achieved as the ratio of $\mathrm{CaCO}_{3}$ to SA reached 3:4. It was reported that the porosity and pore diameter of the beads were increased by increasing the ratio of $\mathrm{CaCO}_{3}$ (Choi et al., 2002). A high proportion of $\mathrm{CaCO}_{3}$ made the microcapsules highly porous and fragile. This was also reflected as an increase in the permeability of the microcapsules. However, the floating ability decreased to $52.38 \%$ when the ratio of $\mathrm{CaCO}_{3}$ to SA increased to $1: 1$. This could be caused by the internal ionotropic gelation effect of $\mathrm{CaCO}_{3}$ on alginate (Choi et al., 2002), as $\mathrm{CaCO}_{3}$ can also be used as a gelling agent to make alginate gel (Kuo and $\mathrm{Ma}, 2001$ ), and the combination of $\mathrm{CaCO}_{3}$ on alginate can enhance the density of the microcapsules. Thus, the optimum ratio of $\mathrm{CaCO}_{3}$ : $\mathrm{SA}$ was $3: 4$.

\section{Concentration of $\mathrm{CaCl}_{2}$}

$\mathrm{CaCl}_{2}$ provides $\mathrm{Ca}^{2+}$ ions for $\mathrm{SA}$ to form hydrogels. The permeability of the microcapsules decreased from 83.03 to $71.45 \%$ with an increase in $\mathrm{CaCl}_{2}$ concentration. The study of $\mathrm{Li}$ et al. (2019) also showed that the increase of $\mathrm{Ca}^{2+}$ concentration reduced the permeability of alginate-based beads. The maximum floating ability (76.19\%) was achieved when the $\mathrm{CaCl}_{2}$ concentration was $3 \%$. With further increase in $\mathrm{Ca}^{2+}$ concentration, the floating ability decreased. It was reported that the gel strength of alginate increased exponentially within a concentration range of $\mathrm{Ca}^{2+}$ (Draget et al., 1993), and the thickness of the external boundary of SA beads increased with the increase of $\mathrm{CaCl}_{2}$ concentration ( $\mathrm{Li}$ et al., 2019). When the concentration of $\mathrm{CaCl}_{2}$ is higher than $3 \%$, the binding sites 
TABLE 1 | Effect of different factors on the properties of the microcapsule (The bold values are for optimized conditions.

\begin{tabular}{|c|c|c|c|c|}
\hline Factor & & Diameter (mm) & Floating ability & Permeability \\
\hline \multirow{5}{*}{$\begin{array}{l}\text { SA concentration } \\
(w / v)\end{array}$} & $1 \%$ & 2.86 & $22.64 \%$ & $84.23 \%$ \\
\hline & $2 \%$ & 3.41 & $50.00 \%$ & $83.89 \%$ \\
\hline & $3 \%$ & 4.10 & $70.83 \%$ & $78.25 \%$ \\
\hline & $4 \%$ & 4.16 & $66.67 \%$ & $73.88 \%$ \\
\hline & $5 \%$ & - & $58.33 \%$ & $71.86 \%$ \\
\hline \multirow{5}{*}{$\begin{array}{l}\text { Mass ratio of } \\
\mathrm{CaCO}_{3}: \mathrm{SA}\end{array}$} & 0 & 3.67 & $6.06 \%$ & $76.12 \%$ \\
\hline & $1: 4$ & 3.67 & $20.00 \%$ & $78.13 \%$ \\
\hline & $1: 2$ & 3.72 & $50.00 \%$ & $78.89 \%$ \\
\hline & $3: 4$ & 3.88 & $70.37 \%$ & $80.02 \%$ \\
\hline & $1: 1$ & 3.88 & $52.38 \%$ & $80.66 \%$ \\
\hline \multirow{5}{*}{$\begin{array}{l}\mathrm{CaCl}_{2} \\
\text { concentration (w/v) }\end{array}$} & $1 \%$ & 3.92 & $37.50 \%$ & $83.03 \%$ \\
\hline & $2 \%$ & 3.96 & $50.00 \%$ & $76.10 \%$ \\
\hline & $3 \%$ & 3.91 & $76.19 \%$ & $75.96 \%$ \\
\hline & $4 \%$ & 3.95 & $68.42 \%$ & $75.22 \%$ \\
\hline & $5 \%$ & 4.00 & $42.11 \%$ & $71.45 \%$ \\
\hline \multirow{5}{*}{$\begin{array}{l}\text { Citric acid } \\
\text { concentration }(\mathrm{w} / \mathrm{v})\end{array}$} & $1 \%$ & 3.56 & $3.85 \%$ & $70.88 \%$ \\
\hline & $2 \%$ & 3.83 & $17.39 \%$ & $73.36 \%$ \\
\hline & $3 \%$ & 4.01 & $69.57 \%$ & $79.21 \%$ \\
\hline & $4 \%$ & 4.07 & $77.27 \%$ & $80.58 \%$ \\
\hline & $5 \%$ & 4.26 & $56.52 \%$ & $84.15 \%$ \\
\hline \multirow{6}{*}{$\begin{array}{l}\text { EC concentration } \\
(w / v)\end{array}$} & 0 & 3.75 & $24.14 \%$ & $69.45 \%$ \\
\hline & $1 \%$ & 3.59 & $80.00 \%$ & $83.27 \%$ \\
\hline & $2 \%$ & 4.06 & $82.76 \%$ & $82.79 \%$ \\
\hline & $3 \%$ & 3.81 & $68.18 \%$ & $84.31 \%$ \\
\hline & $4 \%$ & 4.02 & $65.52 \%$ & $82.91 \%$ \\
\hline & $5 \%$ & 4.11 & $62.96 \%$ & $83.30 \%$ \\
\hline \multirow{5}{*}{$\begin{array}{l}\text { Crosslinking time } \\
\text { (min) }\end{array}$} & 10 & 4.17 & $5.26 \%$ & $69.82 \%$ \\
\hline & 20 & 4.08 & $47.37 \%$ & $71.53 \%$ \\
\hline & 30 & 3.90 & $70.00 \%$ & $77.36 \%$ \\
\hline & 40 & 3.77 & $36.84 \%$ & $62.52 \%$ \\
\hline & 50 & 3.79 & $33.33 \%$ & $61.24 \%$ \\
\hline
\end{tabular}

When conducting the single factor effect experiments, except for the variables, the basic experimental conditions were $3 \% \mathrm{SA}, \mathrm{CaCO}_{3}$ : $\mathrm{SA}$ of $3: 4,3 \% \mathrm{CaCl}_{2}, 3 \%$ citric acid, $2 \%$ EC, and crosslinking for $30 \mathrm{~min})$.

are saturated, resulting in a more compact gel structure, thus increasing the density of the microcapsules.

\section{Concentration of Citric Acid}

Citric acid reacts with $\mathrm{CaCO}_{3}$ to generate $\mathrm{CO}_{2}$ gas which enables the floating of microcapsules. Table 1 shows that the permeability of the microcapsules increased from 70.88 to $84.15 \%$ when the citric acid concentration increased from 1 to $5 \%$. The floating ability increased from 3.85 to $77.27 \%$ as the citric acid concentration increased from 1 to $4 \%$. Citric acid was used as the gas-producing agent in floating microcapsules (Masaki et al., 1991; Soppimath et al., 2001). For example, adding citric acid has successfully delayed the gastric emptying of the calcium alginate beads (Stops et al., 2006) and sustained drug release from the floating pills (Masaki et al., 1991; Ahmed et al., 2016). However, the floating ability decreased to $56.52 \%$ as citric acid concentration increased to $5 \%$. The excess citric acid could react with SA to form alginic acid, thus causing flaccid microstructure in the microcapsules and decrease the floating ability. In this study, $4 \%$ of citric acid was chosen as the optimum concentration.

\section{Concentration of EC}

Ethylcellulose was used as a coating material for the microcapsule. EC is a water-insoluble polymer with a low density and has been studied for the encapsulation and controlled release of drugs (Yang et al., 2001; Prasertmanakit et al., 2009). Furthermore, it can enhance the mechanical strength of the microcapsules and enables effective wrapping of $\mathrm{CO}_{2}$ and thus enhancing the floating ability (Yang and Wang, 2014). Our results showed that microcapsules without EC showed a low floating ability (24.14\%) and relatively low permeability (69.45\%). The highest floating ability (82.76\%) was observed when the EC concentration was $2 \%$ (Table 1), the floating ability decreased as the concentration of EC continuously increased from 2 to $4 \%$. A similar trend was found for the floating SA/HPMC microcapsules in the study of Yang and Wang (2014), where the maximum floating ability was observed with an EC concentration of $30 \mathrm{~g} / \mathrm{L}$. The higher concentration of EC enhanced the matrix density of the microcapsules, thus fewer microcapsules could float. The optimum EC concentration for the microcapsule was $2 \%$.

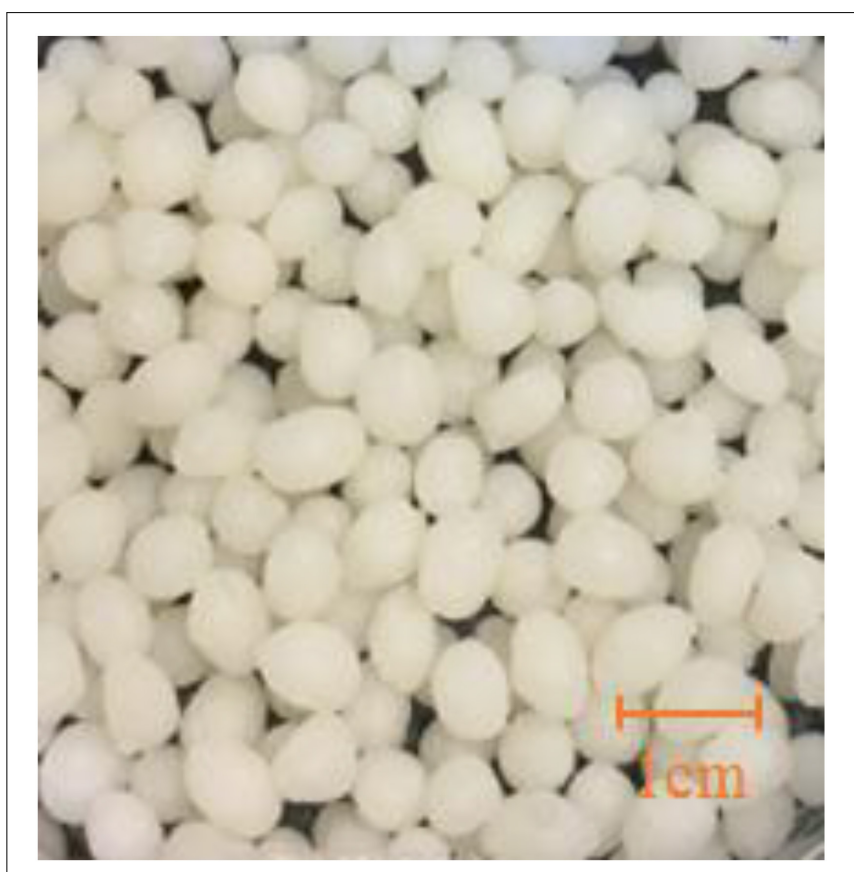

FIGURE 3 | Floating SA-EC microcapsules with algicidal powder as core material, SA as wall material and $\mathrm{EC}$ as the coating material. ( $3 \% \mathrm{SA}, \mathrm{CaCO}_{3}$ : $\mathrm{SA}$ of $3: 4,3 \% \mathrm{CaCl}_{2}, 4 \%$ citric acid, $2 \% \mathrm{EC}$, and crosslinking for $\left.30 \mathrm{~min}\right)$. 


\section{Crosslinking Time}

Gel strength was dependent on the degree of interaction between $\mathrm{SA}$ and $\mathrm{Ca}^{2+}$ ions. The surface microchannel's width of the alginate capsules was also affected by the degree of crosslinking (Li et al., 2019). A sufficient crosslinking time enables the internal gelation of the $\mathrm{Ca}^{2+}$ and SA as well as the reaction of $\mathrm{CaCO}_{3}$ and citric acid, thus enabling the generation of $\mathrm{CO}_{2}$ and the wrapping of $\mathrm{CO}_{2}$ gas. The floating ability increased from 5.26 to $70.00 \%$ as the crosslinking time increased from 10 to $30 \mathrm{~min}$. However, a crosslinking time of $>30 \mathrm{~min}$ could result in the reaction between citric acid and SA to form alginic acid, thus causing a flaccid microstructure in the microcapsules and decrease the floating ability. So a crosslinking time of 30 min was chosen as the optimum.

\section{Optimum Conditions}

Optimum conditions were $3 \% \mathrm{SA}, \mathrm{CaCO}_{3}$ : SA of $3: 4,3 \% \mathrm{CaCl}_{2}$, $4 \%$ citric acid, $2 \% \mathrm{EC}$, and crosslinking for $30 \mathrm{~min}$ (Figure 3 ). The microcapsules had a spherical shape, with complete structure and no cracks on the surface. The volume is about $33 \mathrm{~mm}^{3}$ and with algicidal powder content of $1.7 \mathrm{~g} / \mathrm{L}$.

\section{Evaluation of the Algicidal Activity of the Floating Microcapsules}

The algicidal activity of the microcapsules was tested by coincubating the microcapsules with $A$. sanguinea for up to $24 \mathrm{~h}$. Results showed that the algicidal efficiency of the microcapsules increased significantly ( $p<0.05$ ) over time from $18.71 \pm 1.37 \%$ $(4 \mathrm{~h})$ to $100 \%$ (24 h) (Figure 4), which were significantly higher $(p<0.05)$ than that of the control (microcapsules without algicidal powder). For the control, an algicidal efficiency of $15.44 \pm 0.53 \%$ was observed after $24 \mathrm{~h}$ of incubation. These results indicated that the algicidal powder was slowly released

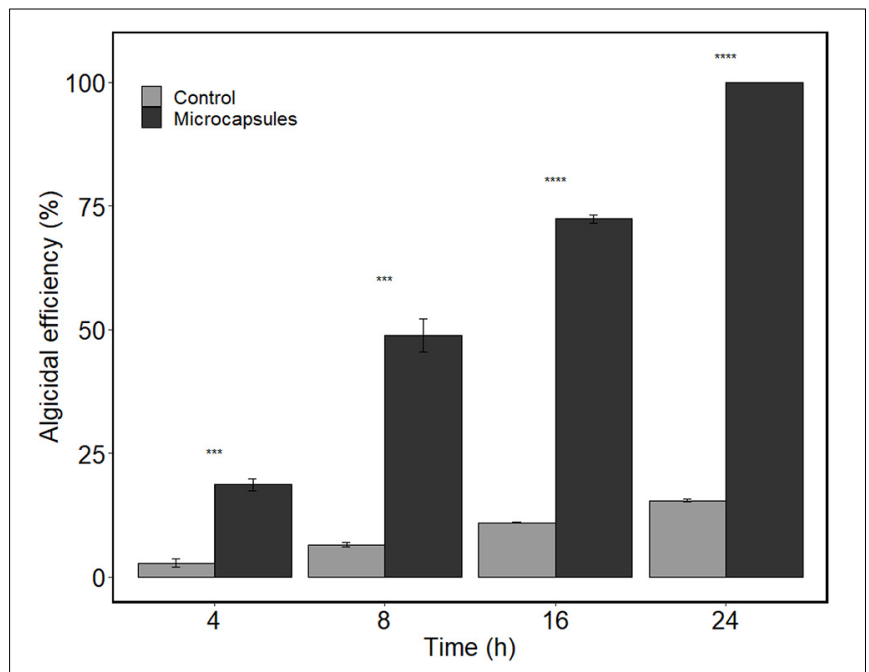

FIGURE 4 | Algicidal efficiency of the floating microcapsules against A. sanguinea. Controls are microcapsules without algicidal powder. Values are

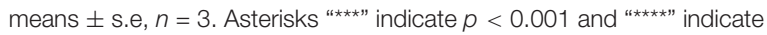
$p<0.0001$.
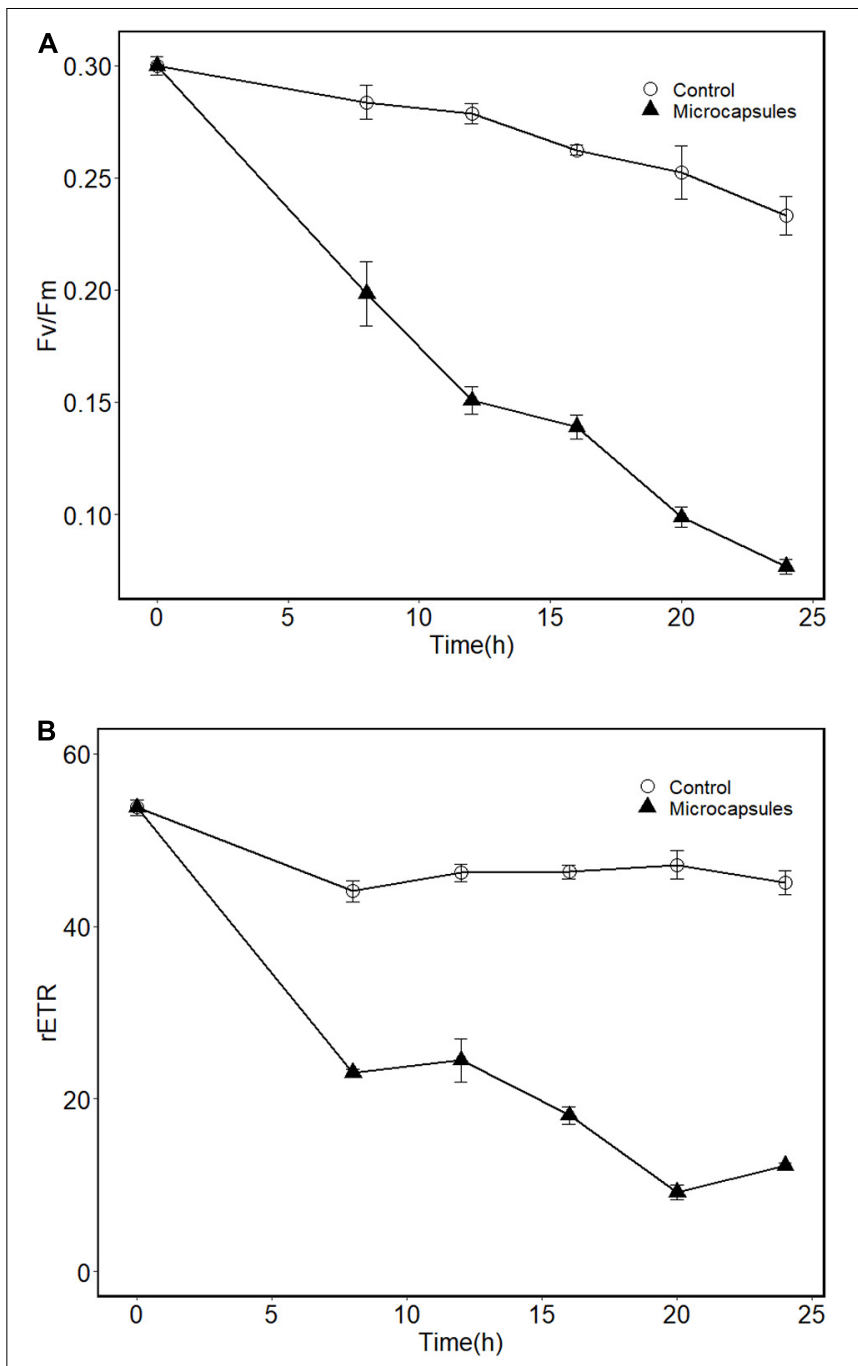

FIGURE 5 | Effects of floating microcapsules on the fluorescence Fv/Fm (A) and rETR (B) of $A$. sanguinea. Controls are microcapsules without algicidal powder. Values are means \pm s.e, $n=3$.

into the algal culture through the pores of the microcapsule and then acted on the algal cells. At present, the immobilization of algicidal bacteria and their potential application in controlling HABs have been studied (Jung et al., 2013; Sun et al., 2015; Wang and Coyne, 2020). To our best knowledge, this is the first report on floating microcapsules based on algicidal powder. In our study, the floating microcapsules achieved a sustained release of the active algicidal compounds and facilitated sufficient contact between the algicidal powder and A. sanguinea.

$\mathrm{Fv} / \mathrm{Fm}$ represents the photochemical efficiency of algal cells, and rETR represents the relative photosynthetic electron transport rate of algal cells. Figure $\mathbf{5}$ shows the changes of Fv/Fm and rETR during incubation. Fv/Fm in control groups experienced significant decreases over time (Figure 5A, $p<0.05)$. For the microcapsules treatment group, the Fv/Fm value decreased significantly from $0.3 \pm 0.007$ to $0.08 \pm 0.005$ $(p<0.05)$. The difference between these two groups was 
significant $(p<0.05)$. The changes of rETR over time during incubation for the control group was not significant (Figure 5B, $p=0.1$. However, for the microcapsules treatment group, rETR decreased significantly over time from $53.76 \pm 1.57$ to $12.27 \pm 0.56(p<0.05)$. The difference between these two groups was significant $(p<0.05)$. It is reported that lower $\mathrm{Fv} / \mathrm{Fm}$ values infer inactivation or down-regulation of PSII reaction centers (Tilney et al., 2014). These results suggested that the addition of floating microcapsules had significant effects on the photosynthetic efficiency and capacity of A. sanguinea (Zhang et al., 2018). A similar phenomenon was observed for the algicidal bacteria Paracoccus sp. Y42 and Pseudoalteromonas S1 when co-incubated with the target algae (Sun et al., 2016; Zhang et al., 2018). Photosynthesis provides primary metabolites and energy for algae. It has been reported that some active algicidal substances inhibit the growth of algae by destroying the photosynthetic pigments, blocking the respiratory chain, and reducing the assimilation products (Tilney et al., 2014; Zhang et al., 2018). Under stress conditions, the photosynthetic apparatus and the transmission of photosynthetic electron of A. sanguinea could be affected (Zhang et al., 2020), thus significant fluctuations in Fv/Fm and rETR values could be observed. The inhibition of both Fv/Fm and rETR indicated that the algicidal compounds released from microcapsules disrupted the photosynthetic apparatus of $A$. sanguinea.

\section{CONCLUSION}

In this study, the algicidal activity of the fermentation broth was significantly improved using repeated batch fermentation of immobilized algicidal bacterium Vibrio brasiliensis H115. Floating microcapsules containing dried fermentation powder was then successfully prepared using SA as wall material, EC as the coating material, and $\mathrm{CaCO}_{3}$ as gas-forming agent. Under the

\section{REFERENCES}

Ahmad, H. A., Ni, S.-Q., Ahmad, S., Zhang, J., Ali, M., Ngo, H. H., et al. (2020). Gel immobilization: a strategy to improve the performance of anaerobic ammonium oxidation (anammox) bacteria for nitrogen-rich wastewater treatment. Bioresour. Technol. 313:123642. doi: 10.1016/j.biortech.2020.123642

Ahmed, S., Ali, A. A., Ali, A. M., and Hassan, O. (2016). Design and in vitro/in vivo evaluation of sussstained-release floating tablets of itopride hydrochloride. Drug Des. Dev. Ther. 2016, 4061-4071. doi: 10.2147/dddt.s115909

Anderson, D. M. (2009). Approaches to monitoring, control and management of harmful algal blooms (HABs). Ocean Coast. Manag. 52, 342-347. doi: 10.1016/ j.ocecoaman.2009.04.006

Choi, B. Y., Park, H. J., Hwang, S. J., and Park, J. B. (2002). Preparation of alginate beads for floating drug delivery system: effects of $\mathrm{CO} 2$ gas-forming agents. Int. J. Pharm. 239, 81-91. doi: 10.1016/s0378-5173(02)00054-6

Draget, K. I., Simensen, M. K., Onsøyen, E., and Smidsrød, O. (1993). Gel strength of Ca-limited alginate gels made in situ. Hydrobiologia 26, 563-565. doi: 10. 1007/978-94-011-1998-6_75

Fukumori, Y., Yamaoka, Y., Ichikawa, H., Fukuda, T., Takeuchi, Y., and Osako, Y. (1988). Coating of pharmaceutical powders by fluidized bed process. II. microcapsules produced by layering of fine powder on coarse particles and subsequent aqueous enteric coating. Chem. Pharm. Bull. 36, 1491-1501. doi: $10.1248 / \mathrm{cpb} .36 .1491$ optimal condition, the microcapsules exhibited a high floating ability and efficient $A$. sanguinea lysis ability by disrupting the photosynthetic apparatus of $A$. sanguinea. These results provide a foundation for the potential application of active algicidal compounds encapsulated in floating microcapsules for controlling A. sanguinea blooms. The purification and identification of the algicidal compounds, as well as the safety evaluation of the microcapsules in marine environment require further investigation.

\section{DATA AVAILABILITY STATEMENT}

The raw data supporting the conclusions of this article will be made available by the authors, without undue reservation.

\section{AUTHOR CONTRIBUTIONS}

LO: formal analysis, writing-original draft. YL: investigation, methodology, and formal analysis. HC: conceptualization and supervision. MZ: writing-review and editing. XY: conceptualization. SW: investigation and methodology. SL: conceptualization, project administration, funding acquisition, and supervision. All authors contributed to the article and approved the submitted version.

\section{FUNDING}

This work was supported by National Key Research and Development Program of China (2020YFD0901003), Shenzhen science and technology application demonstration project (Grant No. KJYY20180201180253571), and Shenzhen Science and Technology Innovation Shenzhen-HongKong Joint Research Project (SGLH20180622152010394).
He, Y., Wu, Z., Tu, L., Han, Y., Zhang, G., and Li, C. (2015). Encapsulation and characterization of slow-release microbial fertilizer from the composites of bentonite and alginate. Appl. Clay Sci. 109-110, 68-75. doi: 10.1016/j.clay.2015. 02.001

Hu, X., Su, H., Xu, Y., Xu, W., Li, S., Huang, X., et al. (2020). Algicidal properties of fermentation products from Bacillus cereus strain JZBC1 dissolving dominant dinoflagellate species Scrippsiella trochoidea, Prorocentrum micans, and Peridinium umbonatum. Biologia 75, 2015-2024. doi: 10.2478/s11756-02000500-3

Idris, A., and Suzana, W. (2005). Effect of sodium alginate concentration, bead diameter, initial $\mathrm{pH}$ and temperature on lactic acid production from pineapple waste using immobilized Lactobacillus delbrueckii. Process Biochem. 41, 11171123. doi: $10.1016 /$ j.procbio.2005.12.002

Jeong, S. Y., Ishida, K., Ito, Y., Okada, S., and Murakami, M. (2003). Bacillamide, a novel algicide from the marine bacterium, Bacillus sp. SY-1, against the harmful dinoflagellate, Cochlodinium polykrikoides. Tetrahedron Lett. 44, 8005-8007. doi: 10.1016/j.tetlet.2003.08.115

Jung, S. W., Kang, Y.-H., Baek, S. H., Lim, D., and Han, M.-S. (2013). Biological control of Stephanodiscus hantzschii (Bacillariophyceae) blooms in a field mesocosm by the immobilized algicidal bacterium Pseudomonas fluorescens HYK0210-SK09. J. Appl. Phycol. 25, 41-50. doi: 10.1007/s10811-012-9836-y

Jung, S. W., Kang, Y.-H., Katano, T., Kim, B.-H., Cho, S.-Y., Lee, J. H., et al. (2010). Testing addition of Pseudomonas fluorescens HYK0210-SK09 to mitigate 
blooms of the diatom Stephanodiscus hantzschii in small- and large-scale mesocosms. J. Appl. Phycol. 22, 409-419. doi: 10.1007/s10811-009-9473-2

Kang, Y. H., Kim, B. R., Choi, H. J., Seo, J. G., Kim, B. H., and Han, M. S. (2007). Enhancement of algicidal activity by immobilization of algicidal bacteria antagonistic to Stephanodiscus hantzschii (Bacillariophyceae). J. Appl. Microbiol. 103, 1983-1994. doi: 10.1111/j.1365-2672.2007. 03439.x

Kang, Y.-H., Jung, S. W., Joo, J.-H., and Han, M.-S. (2012). Use of immobilized algicidal bacteria to control natural freshwater diatom blooms. Hydrobiologia 683, 151-162. doi: 10.1007/s10750-011-0951-6

Kuo, C. K., and Ma, P. X. (2001). Ionically crosslinked alginate hydrogels as scaffolds for tissue engineering: part 1. structure, gelation rate and mechanical properties. Biomaterials 22, 511-521. doi: 10.1016/s0142-9612(00) 00201-5

Lananan, F., Jusoh, A., Ali, N., Lam, S. S., and Endut, A. (2013). Effect of conway medium and $\mathrm{f} / 2$ Medium on the growth of six genera of South China sea marine microalgae. Bioresour. Technol. 141, 75-82. doi: 10.1016/j.biortech.2013.03.006

Li, H.-X., Lu, Z.-M., Geng, Y., Gong, J.-S., Zhang, X.-J., Shi, J.-S., et al. (2015). Efficient production of bioactive metabolites from Antrodia camphorata ATCC 200183 by asexual reproduction-based repeated batch fermentation. Bioresour. Technol. 194, 334-343. doi: 10.1016/j.biortech.2015.06.144

Li, L., Chen, Y., Wang, Y., Shi, F., Nie, Y., Liu, T., et al. (2019). Effects of concentration variation on the physical properties of alginate-based substrates and cell behavior in culture. Int. J. Biol. Macromol. 128, 184-195. doi: 10.1016/ j.ijbiomac.2019.01.123

Li, Y., Liu, L., Xu, Y., Guan, C., Lei, X., Zheng, W., et al. (2016). First evidence of Altererythrobacter sp LY02 with indirect algicidal activity on the toxic dinoflagellate, Alexandrium tamarense. Curr. Microbiol. 73, 550-560. doi: 10. 1007/s00284-016-1093-x

Li, Y., Zhu, H., Lei, X., Zhang, H., Guan, C., Chen, Z., et al. (2015). The first evidence of deinoxanthin from Deinococcus sp. Y35 with strong algicidal effect on the toxic dinoflagellate Alexandrium tamarense. J. Hazard. Mater. 290, 87-95. doi: 10.1016/j.jhazmat.2015.02.070

Lu, X., Zhou, B., Xu, L., Liu, L., Wang, G., Liu, X., et al. (2016). A marine algicidal Thalassospira and its active substance against the harmful algal bloom species Karenia mikimotoi. Appl. Microbiol. Biotechnol. 100, 5131-5139. doi: 10.1007/ s00253-016-7352-8

Ma, F., Sun, Y., Li, A., Zhang, X., and Yang, J. (2015). Activation of accumulated nitrite reduction by immobilized Pseudomonas stutzeri T13 during aerobic denitrification. Bioresour. Technol. 187, 30-36. doi: 10.1016/j.biortech.2015.03. 060

Ma, N., Xu, L., Wang, Q., Zhang, X., Zhang, W., Li, Y., et al. (2008). Development and evaluation of new sustained-release floating microspheres. Int. J. Pharm. 358, 82-90. doi: 10.1016/j.ijpharm.2008.02.024

Masaki, I., Sumio, W., and Yasuo, M. (1991). A new multiple-unit oral floating dosage system. i: preparation and in vitro evaluation of floating and sustainedrelease characteristics. J. Pharm. Sci. 80, 1062-1066. doi: 10.1002/jps. 2600801113

Meleigy, S. A., and Khalaf, M. A. (2009). Biosynthesis of gibberellic acid from milk permeate in repeated batch operation by a mutant Fusarium moniliforme cells immobilized on loofa sponge. Bioresour. Technol. 100, 374-379. doi: 10.1016/j. biortech.2008.06.024

Mori, T., Sato, T., Tosa, T., Matuo, Y., and Chibata, I. (1972). Prepration and characteristics of microcapsules containing asparaginase. Biotechnol. Bioeng. 14, 663-673. doi: 10.1002/bit.260140411

Nochos, A., Douroumis, D., and Bouropoulos, N. (2008). In vitro release of bovine serum albumin from alginate/HPMC hydrogel beads. Carbohydr. Polym. 74, 451-457. doi: 10.1016/j.carbpol.2008.03.020

Porcel, E. M. R., López, J. L. C., Pérez, J. A. S., and Chisti, Y. (2008). Lovastatin production by Aspergillus terreus in a two-staged feeding operation. J. Chem. Technol. Biotechnol. 83, 1236-1243.

Prasertmanakit, S., Praphairaksit, N., Chiangthong, W., and Muangsin, N. (2009). Ethyl cellulose microcapsules for protecting and controlled release of folic acid. AAPS PharmSciTech 10, 1104-1112.

Rani, A. S., Jetty, A., and Ramakrishna, S. V. (2004). Kinetic studies of penicillin production during batch and repeated batch in fluidized bed bioreactor with agar immobilized P. chrysogenum cells. Indian J. Biotechnol. 3, 394-399.
Selvakumaran, S., Muhamad, I. I., and Razak, S. I. A. (2016). Evaluation of kappa carrageenan as potential carrier for floating drug delivery system: effect of pore forming agents. Carbohydr. Polym. 135, 207-214. doi: 10.1016/j.carbpol.2015. 08.051

Soppimath, K. S., Kulkarni, A. R., Rudzinski, W. E., and Aminabhavi, T. M. (2001). Microspheres as floating drug-delivery systems to increase gastric retention of drugs. Drug Metab. Rev. 33, 149-160. doi: 10.1081/dmr-100 104401

Stops, F., Fell, J. T., Collett, J. H., Martini, L. G., Sharma, H. L., and Smith, A. M. (2006). Citric acid prolongs the gastro-retention of a floating dosage form and increases bioavailability of riboflavin in the fasted state. Int. J. Pharm. 308, 14-24. doi: 10.1016/j.ijpharm.2005.09.039

Sun, H.-Y., Zhang, Y., Chen, H.-R., Hu, C.-X., Li, H., and Hu, Z.-L. (2016). Isolation and characterization of the marine algicidal bacterium Pseudoalteromonas S1 against the harmful alga Akashiwo sanguinea. Mar. Biol. 163, 1-8. doi: 10.1016/ j.hal.2014.05.002

Sun, P., Lin, H., Wang, G., Zhang, X., Zhang, Q., and Zhao, Y. (2015). Wheat bran enhances the cytotoxicity of immobilized Alcaligenes aquatilis F8 against Microcystis aeruginosa. PLoS One 10:e0136429. doi: 10.1371/journal.pone. 0136429

Suvase, S. A., Annapure, U. S., and Singhal, R. S. (2010). Gellan gum as an immobilization matrix for the production of cyclosporin a. J. Microbiol. Biotechnol. 20, 1086-1091. doi: 10.4014/jmb.1001. 01006

Tarazona-Janampa, U. I., Cembella, A. D., Pelayo-Zarate, M. C., Pajares, S., Marquez-Valdelamar, L. M., Okolodkov, Y. B., et al. (2020). Associated bacteria and their effects on growth and toxigenicity of the dinoflagellate Prorocentrum lima species complex from epibenthic substrates along mexican coasts. Front. Mar. Sci. 7:569.

Tian, C., Liu, X., Tan, J., Lin, S., Li, D., and Yang, H. (2012). Isolation, identification and characterization of an algicidal bacterium from Lake Taihu and preliminary studies on its algicidal compounds. J. Environ. Sci. 24, 1823-1831. doi: 10.1016/ s1001-0742(11)60983-2

Tilney, C. L., Pokrzywinski, K. L., Coyne, K. J., and Warner, M. E. (2014). Growth, death, and photobiology of dinoflagellates (Dinophyceae) under bacterialalgicide control. J. Appl. Phycol. 26, 2117-2127. doi: 10.1007/s10811-0140248-z

Wang, M., Chen, S., Zhou, W., Yuan, W., and Wang, D. (2020). Algal cell lysis by bacteria: a review and comparison to conventional methods. Algal Res. 46:101794. doi: 10.1016/j.algal.2020.101794

Wang, Y., and Coyne, K. J. (2020). Immobilization of algicidal bacterium Shewanella sp. IRI-160 and its application to control harmful dinoflagellates. Harmful Algae 94:101798. doi: 10.1016/j.hal.2020.101798

Wang, Y., Li, S., Liu, G., Li, X., Yang, Q., Xu, Y., et al. (2020). Continuous production of algicidal compounds against Akashiwo sanguinea via a Vibrio sp. co-culture. Bioresour. Technol. 295:122246. doi: 10.1016/j.biortech.2019. 122246

Wang, Y., Meng, H., Cai, D., Wang, B., Qin, P., Wang, Z., et al. (2016). Improvement of L-lactic acid productivity from sweet sorghum juice by repeated batch fermentation coupled with membrane separation. Bioresour. Technol. 211, 291-297. doi: 10.1016/j.biortech.2016. 03.095

Xu, X., Zhang, W., Gu, X., Guo, Z., Song, J., Zhu, D., et al. (2020). Stabilizing lactate production through repeated batch fermentation of food waste and waste activated sludge. Bioresour. Technol. 300:122709. doi: 10.1016/j.biortech. 2019.122709

Yang, C.-Y., Tsay, S.-Y., and Chen, B.-K. (2001). Application of gelatin for encapsulating aspirin into ethyl cellulose microcapsule in an o/w emulsion. Chem. Eng. Commun. 186, 241-255. doi: 10.1080/00986440108912876

Yang, H., and Wang, J. (2014). Preparation and its performance evaluation of floating microcapsules. Modern Chem. Industry 34, 85-88.

Zhang, B., Cai, G., Wang, H., Li, D., Yang, X., An, X., et al. (2014). Streptomyces alboflavus RPS and its novel and high algicidal activity against harmful algal bloom species Phaeocystis globosa. PLoS One 9:e92907. doi: 10.1371/journal. pone.0092907

Zhang, F., Ye, Q., Chen, Q., Yang, K., Zhang, D., Chen, Z., et al. (2018). Algicidal activity of novel marine bacterium paracoccus sp strain Y42 against a harmful 
algal-bloom-causing dinoflagellate, Prorocentrum donghaiense. Appl. Environ. Microbiol. 84:e01015-18.

Zhang, S.-J., Du, X.-P., Zhu, J.-M., Meng, C.-X., Zhou, J., and Zuo, P. (2020). The complete genome sequence of the algicidal bacterium Bacillus subtilis strain JA and the use of quorum sensing to evaluate its antialgal ability. Biotechnol. Rep. 25:e00421. doi: 10.1016/j.btre.2020.e 00421

Zheng, N., Ding, N., Gao, P., Han, M., Liu, X., Wang, J., et al. (2018). Diverse algicidal bacteria associated with harmful bloom-forming Karenia mikimotoi in estuarine soil and seawater. Sci. Total Environ. 631-632, 1415-1420. doi: 10.1016/j.scitotenv.2018.03.035
Conflict of Interest: The authors declare that the research was conducted in the absence of any commercial or financial relationships that could be construed as a potential conflict of interest.

Copyright (c) 2021 Ouyang, Liu, Chen, Zaynab, Yang, Wang and Li. This is an open-access article distributed under the terms of the Creative Commons Attribution License (CC BY). The use, distribution or reproduction in other forums is permitted, provided the original author(s) and the copyright owner(s) are credited and that the original publication in this journal is cited, in accordance with accepted academic practice. No use, distribution or reproduction is permitted which does not comply with these terms. 